\title{
Influence of Socio-Demographic Characteristics on Academic Performance of Medical Students
}

\author{
Brig Gen (Dr.) Sheikh Salahuddin', Professor Dr. Md. Humayun Kabir Talukder ${ }^{2}$
}

\section{Abstract}

This descriptive cross sectional study was conducted to explore the influence of socio-demographic characteristics on academic performance of medical students. The study period was from July 2015 to June 2016. Total 627 final year MBBS students of 11 (6 government and 5 non-government) medical colleges were selected through convenient sampling technique. A self-administered semi structured questionnaire and an interview schedule were used. Study revealed that family income did not have any influence on the academic performance of medical students. Location of parental stay did not show any influence on students' academic performance. Respondents' previous medium of study had little influence on their academic performance. Students who were regular in passing formative assessment also had better performance in professional examinations. Students who failed in professional examinations mostly failed in oral portions (more than $80 \%$ ). Father's educational qualification did not have any influence on the performance of students but mother's educational qualification had positive relation on it. Performance of those students who were willing to study MBBS were better than unwilling ones. Respondents who were satisfied with the money provided by the parents/guardians were more regular in passing professional examinations. Many of the findings of this study are aligned with findings of the study conducted by Ali $\mathrm{S}$, Haider Z et al. ${ }^{1}$

Key Words: Influence, Socio-demographic characteristics, Academic performance, Medical students

\section{Introduction}

The term "socio-demographic" refers to a group defined by its sociological and demographic characteristics. These characteristics are pertaining to, or characterized by a combination of sociological(related to sociology) and demographic (relating to populations) characteristics. Demographic characteristics can refer to age, sex, and place of residence, religion, educational level and marital status. Sociological characteristics are more objective traits, such as membership in organizations, household status, interests, values and social groups. ${ }^{2}$

A medical student's academic performance attracts the attention of all those involved in medical education. Moreover, the improvement of students' achievements has always been one of the main goals of education. Many studies have stated that the most important predictor of performance in medical school is both prior academic ability and english language proficiency. Socioeconomic status is seen as one of the factors that affect learning; students learn better if they are from an above-average or average income family.

The impact of demographic, socioeconomic and educational patterns on the academic performance is an especially

\footnotetext{
${ }^{2}$ Chief Health Officer, Dhaka South City Corporation

${ }^{3}$ Professor, Curriculum Development \& Evaluation

Centre for Medical Education (CME), Mohakhali, Dhaka.
}

Address of correspondence: Brig Gen (Dr.) Sheikh Salahuddin

Chief Health Officer, Dhaka South City Corporation

E-mail: salahuddin650@yahoo.com relevant issue in developing countries, where major inequality in the distribution of wealth is a serious social concern. ${ }^{4}$

There has been no study on this issue in Bangladesh till date. So, this study would be an effort to explore relationship between the academic performance of medical students and their socio-demographic. These study outcomes may also help recommend medical student selection criteria, widening entry into medical education and develop modalities to improve medical education in particular.

\section{Methodology}

It was a cross-sectional type of descriptive study, conducted in 11 selected government and non-government medical colleges of Bangladesh. Total respondents were 627 conveniently selected final year MBBS students.

Pre tested, self-administered, semi-structured, english questionnaire and interview schedule were used for data collection. Thirty six items were included in the questionnaire and interview schedule included 5 main issues.

Numerical data were processed and analyzed using SPSS software package after proper checking and editing after collection. These data are presented in the form of tables, charts and graphs with necessary descriptions. Qualitative data were presented in descriptive form.

\section{Results}

Eighty eight percent respondents had 'Bangla' as their medium of study in HSC level and $12 \%$ respondents had 'English' as their medium of study in HSC level (Fig 1).

Bangladesh Journal of Medical Education 2017;8(2):18-23. (C) 2017 Salahuddin et al., publisher and licensee Association for Medical Education. This is an Open Access article which permits unrestricted non-commercial use, provided the original work is properly cited. 


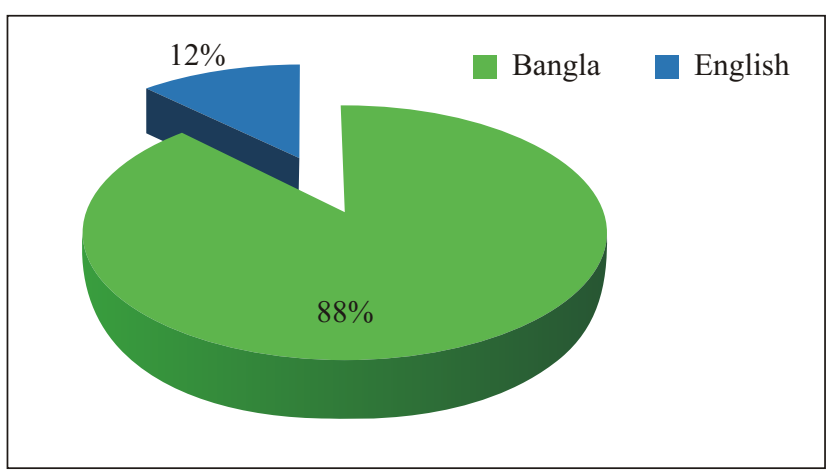

Figure 1. Distribution of respondents by their previous medium of study in HSC

More than eighty one percent respondents of 'Bangla' medium and $90.9 \%$ respondents of 'English' medium passed $1^{\text {st }}$ professional examination in the first chance; while $18.9 \%$ respondents of 'Bangla' medium and 9.1\% respondents passed $1^{\text {st }}$ professional examination with more than one chance (Table 1).

Table 1. Respondents' previous medium of study and regularity in passing $1^{\text {st }}$ professional examination

\begin{tabular}{|c|c|c|c|c|c|c|}
\hline \multirow{2}{*}{$\begin{array}{l}\text { Medium of } \\
\text { study }\end{array}$} & \multicolumn{4}{|c|}{ Chance needed to pass } & \multirow[t]{2}{*}{ Total } & \multirow{2}{*}{$\begin{array}{c}P \\
\text { value }\end{array}$} \\
\hline & $\begin{array}{c}1^{\text {st }} \\
\text { chance }\end{array}$ & $\begin{array}{c}2^{\text {nd }} \\
\text { chance }\end{array}$ & $\begin{array}{c}3^{\text {rd }} \\
\text { chance }\end{array}$ & $\begin{array}{c}>3 \\
\text { chance }\end{array}$ & & \\
\hline Bangla & $\begin{array}{c}446 \\
81.1 \%\end{array}$ & $\begin{array}{c}98 \\
17.8 \%\end{array}$ & $\begin{array}{c}4 \\
.7 \%\end{array}$ & $\begin{array}{c}2 \\
.4 \%\end{array}$ & $\begin{array}{c}550 \\
100 \%\end{array}$ & \multirow{3}{*}{.195} \\
\hline English & $\begin{array}{c}70 \\
90.9 \%\end{array}$ & $\begin{array}{c}7 \\
9.1 \%\end{array}$ & $\begin{array}{c}0 \\
.0 \%\end{array}$ & $\begin{array}{c}0 \\
.0 \%\end{array}$ & $\begin{array}{c}77 \\
100 \%\end{array}$ & \\
\hline Total & $\begin{array}{c}516 \\
82.3 \%\end{array}$ & $\begin{array}{c}105 \\
16.7 \%\end{array}$ & $\begin{array}{c}4 \\
.6 \%\end{array}$ & $\begin{array}{c}2 \\
.3 \%\end{array}$ & $\begin{array}{c}627 \\
100 \%\end{array}$ & \\
\hline
\end{tabular}

Among the respondents 5.6\% from Bangla and 13.0\% from English medium in HSC obtained positions in the merit list of $1^{\text {st }}$ professional examination under respective universities. There is significant difference in obtaining position in the merit list in professional examination between respondents who had 'Bangla' and 'English' medium in HSC level (Table 2).

Table 2. Distribution of respondents by previous medium of study and position they obtained in $1^{\text {st }}$ professional examination under universities

\begin{tabular}{|c|c|c|c|c|}
\hline \multirow[t]{2}{*}{$\begin{array}{l}\text { Medium of study } \\
\text { in HSC }\end{array}$} & \multicolumn{2}{|c|}{$\begin{array}{l}\text { Position in } 1^{\text {st }} \text { professional } \\
\text { examination }\end{array}$} & \multirow[t]{2}{*}{ Total } & \multirow[t]{2}{*}{$\begin{array}{c}P \\
\text { value }\end{array}$} \\
\hline & Yes & No & & \\
\hline Bangla & $\begin{array}{c}31 \\
5.6 \%\end{array}$ & $\begin{array}{c}519 \\
94.4 \%\end{array}$ & $\begin{array}{c}550 \\
100.0 \%\end{array}$ & \multirow{3}{*}{.125} \\
\hline English & $\begin{array}{c}10 \\
13.0 \%\end{array}$ & $\begin{array}{c}67 \\
87.0 \%\end{array}$ & $\begin{array}{c}77 \\
100.0 \%\end{array}$ & \\
\hline Total & $\begin{array}{c}41 \\
6.5 \%\end{array}$ & $\begin{array}{c}586 \\
93.5 \%\end{array}$ & $\begin{array}{c}627 \\
100.0 \%\end{array}$ & \\
\hline
\end{tabular}

Total 554/627 (88.4\%) respondents were from urban colleges and only $11.6 \%$ were from rural colleges. Among the respondents $71.1 \%$ of urban colleges passed $2^{\text {nd }}$ professional examination in 3 years $\& 6$ months. More than seventy two percent respondents from rural college passed $2^{\text {nd }}$ professional examination in 3 years $\& 6$ months (Table 3 ).

Table 3. Location of the college from where respondents passed HSC and attempts needed to pass professional examinations

\begin{tabular}{|l|c|c|c|c|}
\hline \multirow{2}{*}{$\begin{array}{l}\text { Location of } \\
\text { college }\end{array}$} & \multicolumn{2}{|c|}{$\begin{array}{c}\text { Duration needed to pass } \\
\mathbf{2}^{\text {nd }} \text { professional examination }\end{array}$} & \multirow{2}{*}{ Total } & $\begin{array}{c}\text { P } \\
\text { value }\end{array}$ \\
\cline { 2 - 4 } & Y3 yr 6 month & > 3 yr 6 month & & \\
\hline \multirow{2}{*}{ Urban } & 394 & 160 & 554 & \\
& $71.1 \%$ & $28.9 \%$ & $100.0 \%$ & \\
\cline { 1 - 4 } Rural & 53 & 20 & 73 & \multirow{2}{*}{987} \\
\cline { 1 - 3 } Total & $72.6 \%$ & $27.4 \%$ & $100.0 \%$ & \\
& 447 & 180 & 627 & \\
& $71.3 \%$ & $28.7 \%$ & $100.0 \%$ & \\
\hline
\end{tabular}

More than ninety percent respondents who passed formative assessment regularly also passed $2^{\text {nd }}$ professional examination at $1^{\text {st }}$ chance and only $8.9 \%$ respondents who did not pass formative assessment regularly passed $2^{\text {nd }}$ professional examination at $1^{\text {st }}$ chance. There was statistically significant difference in passing professional examination regularity between respondents who passed formative assessment regularly and respondents who did not pass formative assessment regularly (Table 4).

Table 4. Distribution of respondents by regularity in passing formative assessment and $2^{\text {nd }}$ professional examination

\begin{tabular}{|c|c|c|c|c|c|c|}
\hline \multirow{2}{*}{$\begin{array}{l}\text { Formative } \\
\text { assessment } \\
\text { passed } \\
\text { regularly }\end{array}$} & \multicolumn{4}{|c|}{$\begin{array}{l}\text { Attempt to pass professional } \\
\text { examination }\end{array}$} & \multirow[t]{2}{*}{ Total } & \multirow[t]{2}{*}{$\begin{array}{c}P \\
\text { value }\end{array}$} \\
\hline & $\begin{array}{c}1^{\text {st }} \\
\text { chance }\end{array}$ & $\begin{array}{l}2^{\text {nd }} \\
\text { chance }\end{array}$ & $\begin{array}{c}3^{\text {rd }} \\
\text { chance }\end{array}$ & $\begin{array}{c}>3 \\
\text { chance }\end{array}$ & & \\
\hline Yes & $\begin{array}{c}444 \\
90.2 \%\end{array}$ & $\begin{array}{c}47 \\
9.6 \%\end{array}$ & $\begin{array}{c}1 \\
.2 \%\end{array}$ & $\begin{array}{c}0 \\
.0 \%\end{array}$ & $\begin{array}{c}492 \\
100 \%\end{array}$ & \multirow{3}{*}{.000} \\
\hline No & $\begin{array}{c}12 \\
8.9 \%\end{array}$ & $\begin{array}{c}114 \\
84.4 \% \\
\end{array}$ & $\begin{array}{c}6 \\
4.4 \% \\
\end{array}$ & $\begin{array}{c}3 \\
2.2 \%\end{array}$ & $\begin{array}{c}135 \\
100 \%\end{array}$ & \\
\hline Total & $\begin{array}{c}456 \\
72.7 \%\end{array}$ & $\begin{array}{c}161 \\
25.7 \%\end{array}$ & $\begin{array}{c}7 \\
1.1 \%\end{array}$ & $\begin{array}{c}3 \\
.5 \%\end{array}$ & $\begin{array}{c}627 \\
100 \%\end{array}$ & \\
\hline
\end{tabular}

Figure 2 shows the distribution respondents who failed in different portions (written/oral/OSPE or OSCE) in the $2^{\text {nd }}$ professional examination. $81.0 \%$ respondents failed in oral, $10.0 \%$ failed in written and failure in OSPE/OSCE was $9.0 \%$.

Bangladesh Journal of Medical Education 2017;8(2):18-23. 


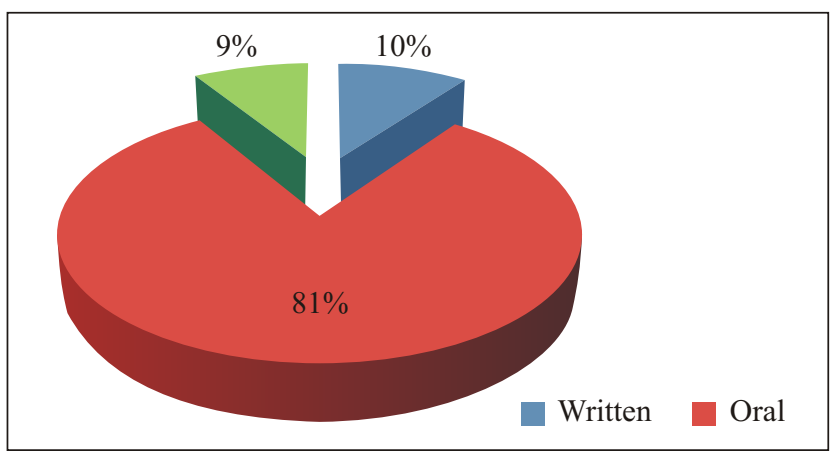

Figure 2. Distribution of respondents who failed in $2^{\text {nd }}$ professional examination by the portion of examination (written/oral/OSPE or OSCE) they failed

Table 5. Distribution of respondents by their family income and regularity in passing professional examination

\begin{tabular}{|c|c|c|c|c|c|c|}
\hline \multirow[t]{2}{*}{$\begin{array}{l}\text { Family income } \\
\text { group }\end{array}$} & \multicolumn{4}{|c|}{$\begin{array}{l}\text { Attempts to pass } \\
1^{\text {st }} \text { professional examination }\end{array}$} & \multirow[t]{2}{*}{ Total } & \multirow[t]{2}{*}{$\begin{array}{c}P \\
\text { value }\end{array}$} \\
\hline & $\begin{array}{c}1^{\text {st }} \\
\text { chance }\end{array}$ & $\begin{array}{c}2^{\text {nd }} \\
\text { chance }\end{array}$ & $\begin{array}{c}3^{\text {rd }} \\
\text { chance }\end{array}$ & $\begin{array}{c}>3 \\
\text { chance }\end{array}$ & & \\
\hline $\begin{array}{l}\text { Low income } \\
\text { (Upto 20000) }\end{array}$ & $\begin{array}{c}81 \\
81.0 \%\end{array}$ & $\begin{array}{c}17 \\
17.0 \%\end{array}$ & $\begin{array}{c}1 \\
1.0 \%\end{array}$ & $\begin{array}{c}1 \\
1.0 \%\end{array}$ & $\begin{array}{c}100 \\
100.0 \%\end{array}$ & \multirow{4}{*}{.584} \\
\hline $\begin{array}{l}\text { Middle income } \\
(20001-50000)\end{array}$ & $\begin{array}{c}268 \\
81.2 \%\end{array}$ & $\begin{array}{c}60 \\
18.2 \%\end{array}$ & $\begin{array}{c}1 \\
.3 \%\end{array}$ & $\begin{array}{c}1 \\
.3 \%\end{array}$ & $\begin{array}{c}330 \\
100.0 \%\end{array}$ & \\
\hline $\begin{array}{l}\text { High income } \\
\text { (Above 50000) }\end{array}$ & $\begin{array}{c}167 \\
84.8 \%\end{array}$ & $\begin{array}{c}28 \\
14.2 \%\end{array}$ & $\begin{array}{c}2 \\
1.0 \%\end{array}$ & $\begin{array}{c}0 \\
.0 \%\end{array}$ & $\begin{array}{c}197 \\
100.0 \%\end{array}$ & \\
\hline Total & $\begin{array}{c}516 \\
82.3 \%\end{array}$ & $\begin{array}{c}105 \\
16.7 \%\end{array}$ & $\begin{array}{c}4 \\
.6 \%\end{array}$ & $\begin{array}{c}2 \\
.3 \%\end{array}$ & $\begin{array}{c}627 \\
100.0 \%\end{array}$ & \\
\hline
\end{tabular}

Table 5 depicts $81.0 \%$ from low income group, $81.2 \%$ from middle income group and $84.8 \%$ from high income group passed $1^{\text {st }}$ professional examination regularly.

Table 6. Location of parental stay and total duration required to pass $2^{\text {nd }}$ professional examination

\begin{tabular}{|c|c|c|c|c|c|c|}
\hline \multirow[t]{2}{*}{$\begin{array}{l}\text { Location of } \\
\text { parental stay }\end{array}$} & \multicolumn{4}{|c|}{$\begin{array}{l}\text { Total duration required to pass } \\
2^{\text {nd }} \text { professional examination }\end{array}$} & \multirow[t]{2}{*}{ Total } & \multirow[t]{2}{*}{$\begin{array}{c}\mathbf{P} \\
\text { value }\end{array}$} \\
\hline & \begin{tabular}{|c|}
$3 \mathrm{yr}$ \\
6 month
\end{tabular} & $4 \mathrm{yr}$ & $\begin{array}{c}4 \mathrm{yr} \\
6 \text { month }\end{array}$ & $\begin{array}{l}5 \text { yr or } \\
\text { more }\end{array}$ & & \\
\hline Dhaka city & $\begin{array}{c}203 \\
70.7 \%\end{array}$ & $\left|\begin{array}{c}70 \\
24.4 \%\end{array}\right|$ & $\begin{array}{c}9 \\
3.1 \%\end{array}$ & $\begin{array}{c}5 \\
1.7 \%\end{array}$ & $\begin{array}{c}287 \\
100.0 \%\end{array}$ & \multirow{7}{*}{.586} \\
\hline $\begin{array}{l}\text { Divisional } \\
\text { town }\end{array}$ & $\begin{array}{c}33 \\
62.3 \% \\
\end{array}$ & $\begin{array}{c}19 \\
35.8 \% \\
\end{array}$ & $1.9 \%$ & $\begin{array}{c}0 \\
.0 \% \\
\end{array}$ & $\begin{array}{c}53 \\
100.0 \% \\
\end{array}$ & \\
\hline District town & $\begin{array}{c}99 \\
70.7 \%\end{array}$ & $\begin{array}{c}35 \\
25.0 \%\end{array}$ & $\begin{array}{c}6 \\
4.3 \%\end{array}$ & $\begin{array}{c}0 \\
.0 \%\end{array}$ & $\begin{array}{c}140 \\
100.0 \%\end{array}$ & \\
\hline $\begin{array}{l}\text { Upazila } \\
\text { proper }\end{array}$ & $\begin{array}{c}66 \\
77.6 \% \\
\end{array}$ & \begin{tabular}{|c|}
17 \\
$20.0 \%$ \\
\end{tabular} & $\begin{array}{c}2 \\
2.4 \% \\
\end{array}$ & $\begin{array}{c}0 \\
.0 \% \\
\end{array}$ & $\begin{array}{c}85 \\
100.0 \% \\
\end{array}$ & \\
\hline $\begin{array}{l}\text { Below } \\
\text { upazila }\end{array}$ & $\begin{array}{c}44 \\
73.3 \%\end{array}$ & \begin{tabular}{c|}
15 \\
$25.0 \%$
\end{tabular} & $\begin{array}{c}0 \\
.0 \%\end{array}$ & $\begin{array}{l}1 \\
1.7 \%\end{array}$ & $\begin{array}{c}60 \\
100.0 \%\end{array}$ & \\
\hline Outside BD & $\begin{array}{c}2 \\
100.0 \% \\
\end{array}$ & $\begin{array}{c}0 \\
0 \% \\
.0 \% \\
\end{array}$ & $\begin{array}{c}0 \\
.0 \% \\
\end{array}$ & $\begin{array}{c}0 \\
.0 \% \\
\end{array}$ & \begin{tabular}{|c|}
2 \\
$100.0 \%$ \\
\end{tabular} & \\
\hline Total & $\begin{array}{c}447 \\
71.3 \%\end{array}$ & $\begin{array}{c}156 \\
24.9 \%\end{array}$ & $\begin{array}{c}18 \\
2.9 \%\end{array}$ & $\begin{array}{c}6 \\
1.0 \%\end{array}$ & $\begin{array}{c}627 \\
100.0 \%\end{array}$ & \\
\hline
\end{tabular}

Table 6 shows $77.6 \%, 73.3 \%, 70.7 \%$ and $62.3 \%$ respondents passed professional examination in the $1^{\text {st }}$ chance whose parents were residing at upazila proper, below upazila, Dhaka city \& district town and divisional town respectively. There was no statistically significant difference in total duration of passing $2^{\text {nd }}$ professional examination among the respondents whose parents were staying in different locations.

Table 7. Educational qualification of father and regularity in passing professional examinations

\begin{tabular}{|c|c|c|c|c|c|c|}
\hline \multirow{2}{*}{$\begin{array}{l}\text { Educational } \\
\text { qualification } \\
\text { of father }\end{array}$} & \multicolumn{4}{|c|}{$\begin{array}{l}\text { Regularity of passing } \\
1^{\text {st }} \text { professional examination }\end{array}$} & \multirow[t]{2}{*}{ Total } & \multirow[t]{2}{*}{$\begin{array}{c}\mathbf{P} \\
\text { value }\end{array}$} \\
\hline & $\begin{array}{c}1^{\text {st }} \\
\text { chance }\end{array}$ & $\begin{array}{c}2^{\text {nd }} \\
\text { chance }\end{array}$ & $\begin{array}{c}3^{\text {rd }} \\
\text { chance }\end{array}$ & $\begin{array}{c}>3 \\
\text { chance }\end{array}$ & & \\
\hline Illiterate & $\begin{array}{c}9 \\
90.0 \%\end{array}$ & $\begin{array}{c}1 \\
10.0 \%\end{array}$ & $\begin{array}{c}0 \\
.0 \%\end{array}$ & $\begin{array}{c}0 \\
.0 \%\end{array}$ & $\begin{array}{c}10 \\
100.0 \%\end{array}$ & \multirow{10}{*}{.097} \\
\hline Pass V & $\begin{array}{c}11 \\
84.6 \%\end{array}$ & $\begin{array}{c}1 \\
7.7 \%\end{array}$ & $\begin{array}{c}0 \\
.0 \%\end{array}$ & $\begin{array}{c}1 \\
7.7 \%\end{array}$ & $\begin{array}{c}13 \\
100.0 \%\end{array}$ & \\
\hline Under SSC & $\begin{array}{c}18 \\
81.8 \%\end{array}$ & $\begin{array}{c}4 \\
18.2 \%\end{array}$ & $\begin{array}{c}0 \\
.0 \%\end{array}$ & $\begin{array}{c}0 \\
.0 \%\end{array}$ & $\begin{array}{c}22 \\
100.0 \%\end{array}$ & \\
\hline SSC & $\begin{array}{c}22 \\
81.5 \%\end{array}$ & $\begin{array}{c}5 \\
18.5 \%\end{array}$ & $\begin{array}{c}0 \\
.0 \%\end{array}$ & $\begin{array}{c}0 \\
.0 \%\end{array}$ & $\begin{array}{c}27 \\
100.0 \%\end{array}$ & \\
\hline HSC & $\begin{array}{c}70 \\
78.7 \%\end{array}$ & $\begin{array}{c}19 \\
21.3 \%\end{array}$ & $\begin{array}{cc}0 & .0 \% \\
2\end{array}$ & $\begin{array}{c}0 \\
.0 \%\end{array}$ & $\begin{array}{c}89 \\
100.0 \%\end{array}$ & \\
\hline $\begin{array}{l}\text { Graduate \& } \\
\text { equivalent }\end{array}$ & $\begin{array}{c}159 \\
78.3 \% \\
\end{array}$ & $\begin{array}{c}42 \\
20.7 \%\end{array}$ & $\begin{array}{c}1.0 \% \\
2\end{array}$ & $\begin{array}{c}0 \\
.0 \% \\
\end{array}$ & $\begin{array}{c}203 \\
100.0 \%\end{array}$ & \\
\hline $\begin{array}{l}\text { Masters \& } \\
\text { equivalent }\end{array}$ & $\begin{array}{c}205 \\
86.1 \%\end{array}$ & $\begin{array}{c}30 \\
12.6 \%\end{array}$ & $\begin{array}{c}.8 \% \\
0\end{array}$ & $\begin{array}{c}1 \\
.4 \% \\
\end{array}$ & $\begin{array}{c}238 \\
100.0 \%\end{array}$ & \\
\hline PhD & $\begin{array}{c}18 \\
90.0 \%\end{array}$ & $\begin{array}{c}2 \\
10.0 \%\end{array}$ & $\begin{array}{c}.0 \% \\
0\end{array}$ & $\begin{array}{c}0 \\
.0 \%\end{array}$ & $\begin{array}{c}20 \\
100.0 \%\end{array}$ & \\
\hline Others & $\begin{array}{c}4 \\
80.0 \%\end{array}$ & $\begin{array}{c}1 \\
20.0 \%\end{array}$ & $\begin{array}{c}100.0 \\
\%\end{array}$ & $\begin{array}{c}0 \\
80.0 \%\end{array}$ & $\begin{array}{c}5 \\
20.0 \%\end{array}$ & \\
\hline Total & $\begin{array}{c}516 \\
82.3 \%\end{array}$ & $\begin{array}{c}105 \\
16.7 \%\end{array}$ & $\begin{array}{c}4 \\
.6 \%\end{array}$ & $\begin{array}{c}2 \\
.3 \%\end{array}$ & $\begin{array}{c}627 \\
100.0 \%\end{array}$ & \\
\hline
\end{tabular}

Table 7 shows $90.0 \%$ respondents of both illiterate fathers and $\mathrm{PhD}$ qualified fathers passed $1^{\text {st }}$ professional examination at the $1^{\text {st }}$ attempt. There was no statistically significant difference in regularity of passing $1^{\text {st }}$ professional examination and educational qualification the respondents' fathers.

Bangladesh Journal of Medical Education 2017;8(2):18-23. 
Table 8. Educational qualification of mother and regularity in passing professional examinations

\begin{tabular}{|c|c|c|c|c|c|c|}
\hline \multirow{2}{*}{$\begin{array}{l}\text { Educational } \\
\text { qualification } \\
\text { of mother }\end{array}$} & \multicolumn{4}{|c|}{$\begin{array}{l}\text { Regularity of passing } 1^{\text {st }} \\
\text { professional examination }\end{array}$} & \multirow[t]{2}{*}{ Total } & \multirow[t]{2}{*}{$\begin{array}{c}P \\
\text { value }\end{array}$} \\
\hline & $\begin{array}{c}1^{\text {st }} \\
\text { chance }\end{array}$ & $\begin{array}{c}2^{\text {nd }} \\
\text { chance }\end{array}$ & $\begin{array}{c}3^{\text {rd }} \\
\text { chance }\end{array}$ & $\begin{array}{c}>3 \\
\text { chance }\end{array}$ & & \\
\hline Illiterate & $\begin{array}{c}10 \\
71.4 \% \\
\end{array}$ & $\begin{array}{c}3 \\
21.4 \% \\
\end{array}$ & $\begin{array}{c}0 \\
.0 \%\end{array}$ & $\begin{array}{c}1 \\
7.1 \%\end{array}$ & $\begin{array}{c}14 \\
100.0 \%\end{array}$ & \\
\hline Pass V & $\begin{array}{c}21 \\
91.3 \%\end{array}$ & $\begin{array}{c}2 \\
8.7 \%\end{array}$ & $\begin{array}{c}0 \\
.0 \%\end{array}$ & $\begin{array}{c}0 \\
.0 \%\end{array}$ & $\begin{array}{c}23 \\
100.0 \%\end{array}$ & \\
\hline Under SSC & $\begin{array}{c}54 \\
80.6 \%\end{array}$ & $\begin{array}{c}13 \\
19.4 \%\end{array}$ & $\begin{array}{c}0 \\
.0 \%\end{array}$ & $\begin{array}{c}0 \\
.0 \%\end{array}$ & $\begin{array}{c}67 \\
100.0 \%\end{array}$ & \\
\hline SSC & $\begin{array}{c}97 \\
85.1 \%\end{array}$ & $\begin{array}{c}15 \\
13.2 \%\end{array}$ & $\begin{array}{c}2 \\
1.8 \%\end{array}$ & $\begin{array}{c}0 \\
.0 \%\end{array}$ & $\begin{array}{c}114 \\
100.0 \%\end{array}$ & \\
\hline HSC & $\begin{array}{c}109 \\
79.0 \%\end{array}$ & $\begin{array}{c}27 \\
19.6 \%\end{array}$ & $\begin{array}{c}2 \\
1.4 \%\end{array}$ & $\begin{array}{c}0 \\
.0 \%\end{array}$ & $\begin{array}{c}138 \\
100.0 \%\end{array}$ & .029 \\
\hline $\begin{array}{l}\text { Graduate \& } \\
\text { equivalent }\end{array}$ & $\begin{array}{c}129 \\
81.1 \%\end{array}$ & $\begin{array}{c}30 \\
18.9 \%\end{array}$ & $\begin{array}{c}0 \\
.0 \%\end{array}$ & $\begin{array}{c}0 \\
.0 \%\end{array}$ & $\begin{array}{c}159 \\
100.0 \%\end{array}$ & \\
\hline $\begin{array}{l}\text { Masters \& } \\
\text { equivalent }\end{array}$ & $\begin{array}{c}88 \\
86.3 \%\end{array}$ & $\begin{array}{c}13 \\
12.7 \%\end{array}$ & $\begin{array}{c}0 \\
.0 \%\end{array}$ & $\begin{array}{c}1 \\
1.0 \%\end{array}$ & $\begin{array}{c}102 \\
100.0 \%\end{array}$ & \\
\hline PhD & $\begin{array}{c}8 \\
80.0 \%\end{array}$ & $\begin{array}{c}2 \\
20.0 \%\end{array}$ & $\begin{array}{c}0 \\
.0 \%\end{array}$ & $\begin{array}{c}0 \\
.0 \%\end{array}$ & $\begin{array}{c}10 \\
100.0 \%\end{array}$ & \\
\hline Total & $\begin{array}{c}516 \\
82.3 \%\end{array}$ & $\begin{array}{c}105 \\
16.7 \%\end{array}$ & $\begin{array}{c}4 \\
.6 \%\end{array}$ & $\begin{array}{c}2 \\
.3 \%\end{array}$ & $\begin{array}{c}627 \\
100.0 \%\end{array}$ & \\
\hline
\end{tabular}

Table 8 shows $91.3 \%$ respondents who had class $\mathrm{V}$ pass mothers and masters \& equivalent degree holder mothers passed $1^{\text {st }}$ professional examination at the $1^{\text {st }}$ attempt. There was statistically significant difference in regularity of passing $1^{\text {st }}$ professional examination and the educational qualification of respondents' mothers.

Table 9. Respondents' willingness to study MBBS course and total period required to pass $2^{\text {nd }}$ professional examination

\begin{tabular}{|c|c|c|c|c|c|c|}
\hline \multirow{2}{*}{$\begin{array}{l}\text { Willingness } \\
\text { to study } \\
\text { MBBS }\end{array}$} & \multicolumn{4}{|c|}{$\begin{array}{l}\text { Duration required to pass } 2^{\text {nd }} \\
\text { professional examination }\end{array}$} & \multirow[t]{2}{*}{ Total } & \multirow[t]{2}{*}{$\begin{array}{c}P \\
\text { value }\end{array}$} \\
\hline & $\begin{array}{l}3 \text { yr } 6 \\
\text { month }\end{array}$ & $4 \mathrm{yr}$ & $\begin{array}{l}4 \text { yr } 6 \\
\text { month }\end{array}$ & $\begin{array}{l}>4 \text { yr } 6 \\
\text { month }\end{array}$ & & \\
\hline Yes & $\begin{array}{c}389 \\
75.0 \%\end{array}$ & $\begin{array}{c}115 \\
22.2 \%\end{array}$ & $\begin{array}{c}13 \\
2.5 \%\end{array}$ & $\begin{array}{c}2 \\
.4 \% \\
\end{array}$ & $\begin{array}{c}519 \\
100.0 \%\end{array}$ & \multirow{3}{*}{.000} \\
\hline No & $\begin{array}{c}58 \\
53.7 \%\end{array}$ & $\begin{array}{c}41 \\
38.0 \%\end{array}$ & $\begin{array}{c}5 \\
4.6 \%\end{array}$ & $\begin{array}{c}4 \\
3.7 \%\end{array}$ & $\begin{array}{c}108 \\
100.0 \%\end{array}$ & \\
\hline Total & $\begin{array}{c}447 \\
71.3 \%\end{array}$ & $\begin{array}{c}156 \\
24.9 \%\end{array}$ & $\begin{array}{c}18 \\
2.9 \%\end{array}$ & $\begin{array}{c}6 \\
1.0 \%\end{array}$ & $\begin{array}{c}627 \\
100.0 \%\end{array}$ & \\
\hline
\end{tabular}

Table 9 shows $75.0 \%$ respondents who were willing and $53.7 \%$ respondents who were unwilling to study MBBS passed $2^{\text {nd }}$ professional examination at the shortest possible duration i.e. 3 year 6 month. There was statistically significant difference in total duration of passing $2^{\text {nd }}$ professional examination and the willingness of respondents. Students who were willing to study MBBS took less duration in passing professional examinations.
Table 10. Satisfaction on money provided by parents/guardians to respondents and total duration needed to pass $2^{\text {nd }}$ professional examination

\begin{tabular}{|c|c|c|c|c|c|c|}
\hline \multirow{2}{*}{$\begin{array}{l}\text { Satisfaction } \\
\text { on money } \\
\text { provided } \\
\text { by parents/ } \\
\text { guardians }\end{array}$} & \multicolumn{4}{|c|}{$\begin{array}{l}\text { Duration required to pass } 2^{\text {nd }} \\
\text { professional examination }\end{array}$} & \multirow[t]{2}{*}{ Total } & \multirow[t]{2}{*}{$\begin{array}{c}P \\
\text { value }\end{array}$} \\
\hline & $\begin{array}{l}3 \text { yr } 6 \\
\text { month }\end{array}$ & $4 \mathrm{yr}$ & $\begin{array}{l}4 \text { yr } 6 \\
\text { month }\end{array}$ & $\begin{array}{l}>4 \text { yr } 6 \\
\text { month }\end{array}$ & & \\
\hline Yes & $\begin{array}{c}374 \\
73.3 \%\end{array}$ & $\begin{array}{c}120 \\
23.5 \%\end{array}$ & $\begin{array}{c}13 \\
2.5 \%\end{array}$ & $\begin{array}{c}3 \\
.6 \%\end{array}$ & $\begin{array}{c}510 \\
100.0 \% \\
\end{array}$ & \multirow{3}{*}{.037} \\
\hline No & $\begin{array}{r}73 \\
62.4 \% \\
\end{array}$ & $\begin{array}{c}36 \\
30.8 \% \\
\end{array}$ & $\begin{array}{l}5 \\
4.3 \% \\
\end{array}$ & $\begin{array}{c}3 \\
2.6 \% \\
\end{array}$ & $\begin{array}{c}117 \\
100.0 \% \\
\end{array}$ & \\
\hline Total & $\begin{array}{c}447 \\
71.3 \%\end{array}$ & $\begin{array}{c}156 \\
24.9 \%\end{array}$ & $\begin{array}{c}18 \\
2.9 \%\end{array}$ & $\begin{array}{c}6 \\
1.0 \%\end{array}$ & $\begin{array}{c}627 \\
100.0 \%\end{array}$ & \\
\hline
\end{tabular}

Among the respondents $73.3 \%$ who were satisfied with the money provided by their parents/guardians passed $2^{\text {nd }}$ professional examination at the shortest possible duration i.e. 3 year 6 month. Whereas, $62.4 \%$ respondents who were unsatisfied with the money provided by their parents/guardians passed $2^{\text {nd }}$ professional examination at the shortest duration. There was statistically significant difference in total duration of passing $2^{\text {nd }}$ professional examination and the money provided by the parents/guardians of respondents. Satisfied respondents were more regular in passing $2^{\text {nd }}$ professional examination.

\section{Discussion}

It is a challenge to identify socio-demographic factors that can influence academic performance of medical students. This study was undertaken to explore the influence of sociodemographic characteristics on academic performance among 627 final year MBBS students of 11selected government and non-government medical colleges of Bangladesh. Data was collected from the respondents using semi-structured self-administered questionnaire. Interview schedule was also used for in-depth interview to probe the obscured issues for selected participants.

We found eighty eight percent respondents had 'Bangla' as their medium of study in HSC level and $12 \%$ respondents had 'English' as their medium of study in HSC level. It is more or less consistent with the study conducted by Ali S, Haider $\mathrm{Z}$ et al. ${ }^{1}$ There is no significant difference in the attempt of passing in $1^{\text {st }}$ professional examination between respondents who had 'Bangla' or 'English' medium in HSC (Table 1). There is significant difference in obtaining position in the merit list of professional examination between respondents who had 'Bangla' and 'English' medium in HSC level (Table 2). Academic performance of graduate students with respect their medium of instruction in school (i.e. English/Urdu) is equal. Another study stated that academic performance of graduate students vary with medium of schooling. ${ }^{1,5}$

Total $88.4 \%$ respondents were from urban colleges and only $11.6 \%$ were from rural colleges. Findings of this study are more or less consistent with the study conducted by Ali S, 
Haider $\mathrm{Z}$ et al. ${ }^{1}$ where $84 \%$ graduate students belong to urban area and $16 \%$ belong to rural area of a university. Among the respondents $71.1 \%$ from urban and $72.6 \%$ respondents from rural (intermediate) colleges passed $2^{\text {nd }}$ professional examination in 3 years \& 6 months (Table 3 ). Findings of this study are consistent to the study conducted by Miller WP, Birch RE et al. ${ }^{5}$ where they concluded, on average academic performance of graduate students with respect to their residential area are equal.

Among the respondents who failed in different portions (written/oral/OSPE or OSCE) in the $2^{\text {nd }}$ professional examination, $81.0 \%$ respondents failed in oral, $10.0 \%$ failed in written and failure in OSPE/OSCE was $9.0 \%$ (Fig 2). Maximum failure in oral $(81 \%)$ in $2^{\text {nd }}$ professional examinations may be due to improper/non-use of structured oral examination system.

Above ninety percent respondents who passed formative assessment regularly also passed $2^{\text {nd }}$ professional examination at $1^{\text {st }}$ chance. Most of the students who regularly passed formative assessment also passed professional examinations regularly (Table 4). Around sixty nine percent teachers agreed/strongly agreed that formative assessment encourage student's deep learning. This study also revealed that formative assessment has got significant effect on summative assessment on various aspects. ${ }^{6}$

Eighty one percent respondents from low income group, $81.2 \%$ from middle income group and $84.8 \%$ from high income group passed $1^{\text {st }}$ professional examination regularly (Table 5). A Study conducted in India showed no linkage of socio-economic status with academic performance of the students ${ }^{7}$ which has similarity with this study. Study of Peter Oyier Ogweno, Nephat et al. ${ }^{8}$ concluded that low family earnings did not influence performance of students in agriculture.

Among the respondents $77.6 \%, 73.3 \%, 70.7 \%$ and $62.3 \%$ respondents passed $2^{\text {nd }}$ professional in 3 year 6 months whose parents were residing at upazila proper, below upazila, Dhaka city \& district town and of divisional town respectively (Table 6). Findings of this study are consistent to a study which stated that average academic performance of graduate students with respect to their residential area (Urban/Rural) are equal. ${ }^{5}$

Ninety percent respondents of both illiterate and $\mathrm{PhD}$ qualified fathers passed $1^{\text {st }}$ professional examination at the $1^{\text {st }}$ attempt. There was no statistically significant difference in regularity of passing $1^{\text {st }}$ professional examination and educational qualification of respondents' fathers. More than ninety one percent respondents who had class $\mathrm{V}$ pass mothers and masters \& equivalent degree holder mothers passed $1^{\text {st }}$ professional examination at the $1^{\text {st }}$ attempt. There was statistically significant difference in regularity of passing $1^{\text {st }}$ professional examination and the educational qualification of respondents' mothers (Table $7 \& 8$ ). There is a positive correlation between level of education of the parents, mother in particular and the students' performance. Parental low levels of education positively influenced students' performance. ${ }^{8}$ Findings of this study are consistent with the mentioned study.

Seventy five percent respondents who were willing and $53.7 \%$ respondents who were unwilling to study MBBS passed $2^{\text {nd }}$ professional examination at the shortest possible duration i.e. 3 year 6 month. Students who were willing to study MBBS required less duration in passing professional examinations (Table 9). Poor performance of medical students was primarily due to stress, low socio-economic status and their disinterest in studies. ${ }^{9}$

Among the respondents $73.3 \%$ who were satisfied with the money provided by their parents/guardians passed $2^{\text {nd }}$ professional examination at the shortest possible duration i.e. 3 year 6 month. Satisfied respondents were more regular in passing $2^{\text {nd }}$ professional examination (Table 10). There is correlation between parents' socio-economic status on students' academic achievement. Poor performance of medical students was primarily due to stress, low socio-also economic status and their disinterest in studies. ${ }^{9-10}$

\section{Conclusion}

In this highly competitive world, good academic performance has become a key to student's future. Achieving high academic achievement is one of the most important goals of education. This descriptive cross sectional study investigates the influence of socio-demographic characteristics on academic performance of final year MBBS students of selected medical colleges. The findings of this study suggest that few socio-demographic factors play role in the achievement of good academic performance. The main factors which affect the academic performance of medical students are inadequate proficiency in English language, mother's educational qualification, adequacy of money provided by the parents/guardians to the students and willingness of students to study medical science.

Further research on randomly selected sample using more time and resources in this issue is needed on the basis of which findings can be generalized.

\section{References}

1. Ali S, Haider Z, Munir F, Khan H, Ahmed A. Factors Contributing to the Students' Academic Performance: A Case Study of Islamia University Sub-Campus. American Journal of Educational Research 2013; 1(8): pp. 283-289.

2. Http://www.ask.com/world-view/sociodemographic mean-cc3b7119d0e7aefe date 28 December 2015.

3. Shawwa LA, Ahmad AA, Rhman A, Merdad A, Sara B, Algethami A, Abu-shanab J, \& Balkhoyor AR. Factors potentially influencing academic performance among medical students. Adv Med Educ Pract 2015; 6: pp. 6575.

4. Azhar M, NadeemS, Naz F, Perveen F \& Sameen A.

Bangladesh Journal of Medical Education 2017;8(2):18-23. 
Impact of parental education and socioeconomic status on academic achievements on university students. International Journal of Academic Research and Reflection 2013; 1(3).

5. Miller WP and Birch RE. The influence of type of high school attended on university performance. Retrieved on December 3, 2008 from http://www3.interscience. wiley.com/journal/118538313.

6. Hossain S, Begum N, Talukder HK. Teachers' evaluation of formative assessment on summative assessment in undergraduate medical education. Bangladesh Journal of Medical Education 2012; 03(01): pp. 18-21.

7. Mondal A, Ghosh A, Sengupta G, Bera T, Das N, Mukharjee. Factors affecting the performance of undergraduate medical students: a perspective. Indian J Community Med 2012;37(2): 126-129.
8. Ogweno PO, Nephat, JK, Obara J. Influence of Family Characteristics on Academic Performance of Students in Secondary Agriculture, in Rachuonyo North Sub County, Kenya. International Journal of Education and Research 2014;2(2): pp. 1-12.

9. Sultana A, Shaikh AR, Chaudhry AS. Discrepancy of Academic Performance Between High School and Professional Results of Medical Students. Journal of Rawalpindi Medical College 2013; 17(2): PP. 288-290.

10. Nora AZ, Thabet AM and Asmaa KH. Effect of peer group and parents' socioeconomic status on academic achievement among preparatory schools students at Assuit City. Al-Azhar Assuit Medical Journal 2014; 12(1): pp. 309-332.

Bangladesh Journal of Medical Education 2017;8(2):18-23. 